\title{
A Study on the Detachment Characteristics of the Tramwave Catenary-free Electrification System for Urban Traffic
}

\author{
Song Xiao, Can Zhang, Yuanpei Luo, Jingchi Wu, Yang Rao, and J. K. Sykulski, Fellow, IEEE
}

\begin{abstract}
The Tramwave Catenary-free System (TCS) - a novel type of electrified transit system for urban railways receives traction energy from a flexible ground-embedded ferromagnetic strip, which is energized only if a corresponding segment is located underneath the vehicle body. The current collector in the TCS is equipped with an 'I-shaped' permanent magnet combination that produces a lifting force on the flexible ferromagnetic strips with octopus-shaped copper sheets located on the surface of the strips underneath the ground. The vehicle's high-voltage circuit conducts when an electrical contact is maintained by the attractive force of the permanent magnet combination mounted inside the magnetic current collector. However, it has recently been observed that due to the dynamic processes during the electrical contact a severe electrochemical corrosion may occur on the surface of the octopus-shaped copper sheets, which can jeopardize the safe operation of the whole electrified transit system of trams. In order to identify the origins of this electrochemical corrosion, the dynamic characteristics of the TCS have been evaluated with particular attention paid to the impact of eddy currents on the attractive force of the permanent magnet combination under variable operational velocities of vehicles. Based on the dynamic characteristics of TCS, the arc erosion process on the surface of the copper sheet has been reproduced using an arc-discharge experimental platform and the causes of electrochemical corrosion in the TCS have been found. The damage development trends - under increasing speeds of trams - evaluated by the dynamic model of the TCS are consistent with the statistical data for practical damage situations.
\end{abstract}

Index Terms-Electrified transit system, Tramwave Catenaryfree system, permanent magnet current collector, electrical contact, electrochemical corrosion, dynamic characteristics.

\footnotetext{
Manuscript received February 15, 2020; revised ? ??, 2020; accepted ? ??. Date of publication ?? ??, 2020; date of current version ?? ??, 2020.This work was supported in part by the National Natural Science Foundation for Distinguished Young Scholars of China under Grant 51707166, and in part by the Scientific research project of Central University Grant 2682018CX16 as well as the Sichuan Science and Technology General Project Grant 2019YJ0213. The review of this paper was arranged by Senior Editor ??. ????. (Corresponding author: Song Xiao).

S. Xiao, C. Zhang, Y. Luo, J. Wu, Y. Rao are with the School of Electrical Engineering, Southwest Jiaotong University, Chengdu 610031, China (e-mail: xiaosong@home.swjtu.edu.cn; CanZhang@my.swjtu.edu.cn; ypluo@my.swjtu.edu.cn; jingchiwu@my.swjtu.edu.cn; ry@my.swjtu.edu. $\mathrm{cn})$.

J. K. Sykulski is with Electronics and Computer Science, University of Southampton, SO17 1BJ, UK (e-mail: jks@soton.ac.uk).

Digital Object Identifier (inserted by IEEE).
}

\section{INTRODUCTION}

$\mathrm{U}$ RBAN public transport vehicles, such as trams, metros and maglev trains, play a critical role in reducing traffic congestion in cities with high population density all over the world. But the design of electrified mass transit systems for urban railway traffic is often a complicated task, because many elements such as safety, efficiency, cost and even appearance must be considered. The use of traditional electrified transit systems based on electrical flexible/rigid contact wires (catenary or active rails) is usually restricted by the safetyrelated constraints [1], [2]. When catenary systems are chosen [3], special corridors must normally be constructed to ensure that no other vehicle or person can approach the high-voltage contact lines, leading to more space usage and obvious visual impact.

The Tramwave catenary-free system (TCS) shown in Fig. 1 is a highly efficient power supply system for railway vehicles such as trams or light metros. It receives traction energy from flexible ferromagnetic strips buried underneath the ground and allows space sharing with urban street traffic and pedestrians [1]. The traction energy is transferred to trams via an electrical contact between the magnetic current collector and the conducting ferromagnetic strip; only a segment located underneath the tram's body is energized, while the remaining parts of the supplying line are free from electrical voltage (Fig. 2). This catenary-free electrified transit system has numerous advantages such as safe operation, low electromagnetic radiation, little space occupancy, good visual design, etc.

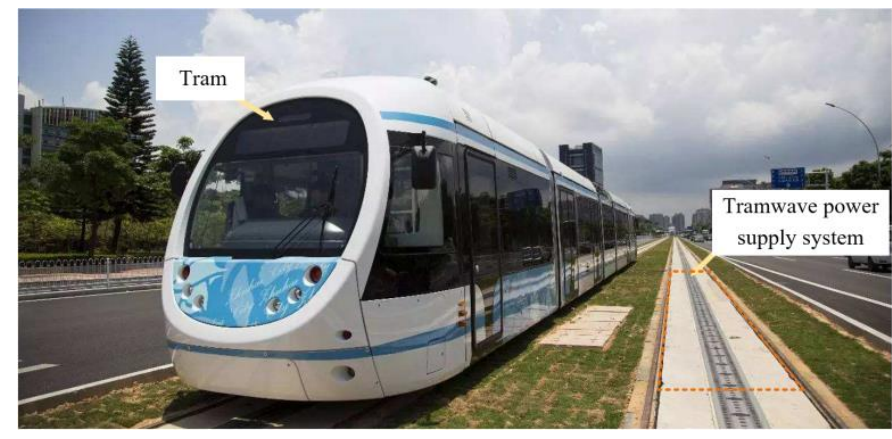

Fig. 1. A picture of a catenary-free urban railway.

However, the operating experience of the existing tram railways using TCS reveals that some serious electrochemical corrosion damage can take place in the ground power supply 
modules, thus directly affecting the quality and efficiency of the power transmission. A similar arc discharge also occurs in the pantograph-catenary system (PCS) of high-speed trains because of off-line cases when passing the suspension points distributed along the contact wire especially under high-speed conditions [5]-[7]. The main reasons for poor electrical contact between the pantograph strips and the contact wire include unsmooth contact wire, irregular rails and vibration of the contact wire produced by environmental elements. Some dynamic models of pantograph-catenary system have been developed to investigate the thermal and electrical performance of pantograph strips during motion [8]-[11]. But there are important differences between the PCS and the TCS operation. The TCS is a sectional power supply system operating under two alternative conditions: connected or disconnected. Moreover, the electrical contact between the ferromagnetic strip and the magnetic current collector in TCS is maintained by a magnetic force varying with the tram speed, rather than the constant lift driven by air pressure in PCS. With the increasing speed, the magnetic force may be influenced by eddy currents in the ferromagnetic strips or other external elements. Until now, little research has been done to identify the origins causing the electrochemical corrosion in the TCS, although some previous work has been undertaken to analyze the varying force conditions between permanent magnets and the ferromagnetic materials [12], [13], which provides the basis for examining the reasons for damage occurring in TCSs.

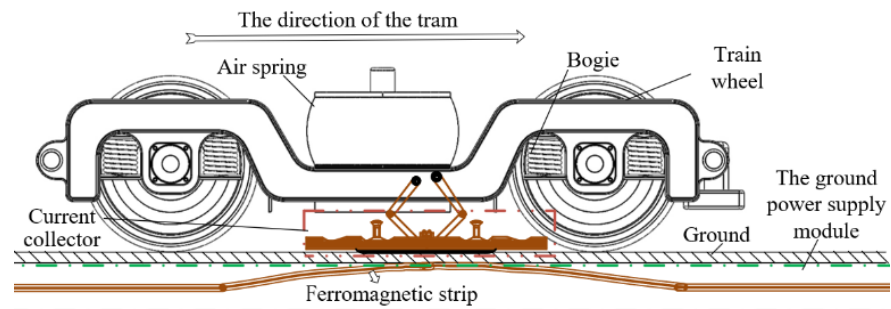

Fig. 2. The configuration of the bogie with the magnetic current collector used to house the underground flexible steel band.

In order to analyze the impact brought by the eddy current effect on the magnetic force, the variation of magnetic force produced by the permanent magnetic current collector is first examined as a function of the speed of the tram. The variation trends are then incorporated into the dynamic model of the TCS for evaluating the dynamic electrical contact process. Finally, on the basis of the analysis of the dynamic process, the reproduction of the electrochemical corrosion damage under different tram speeds is attempted with the help of the arc discharge experiments.

\section{The OPERATING PRINCIPLE OF THE TCS}

In order to facilitate the understanding of the operating principle of the TCS, its cross section is presented in Fig. 3. The ground power supply system is composed of several critical components including conductive plates, octopusshape copper sheets, positive brushes, negative brushes and ferromagnetic strips. The permanent magnet current collector is positioned at the bottom of the vehicle body.
When the ferromagnetic strip with copper sheets is raised by the attractive force produced by the magnetic current collector, the conducting TCS transfers power from the ground supply module to the electric traction and control system on the tram.

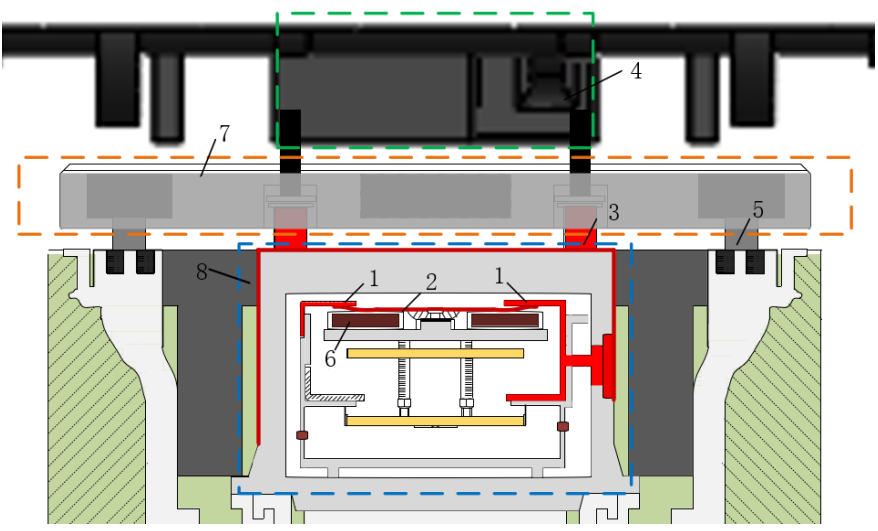

Fig. 3. The cross section of the TCS with the critical components:

1. Conductive plate, 2. Octopus-shape copper sheet, 3. Positive carbon brush, 4. Traction system, 5. Negative carbon brush, 6. Ferromagnetic strip, 7. Current collector, 8 . Ground power supply module.

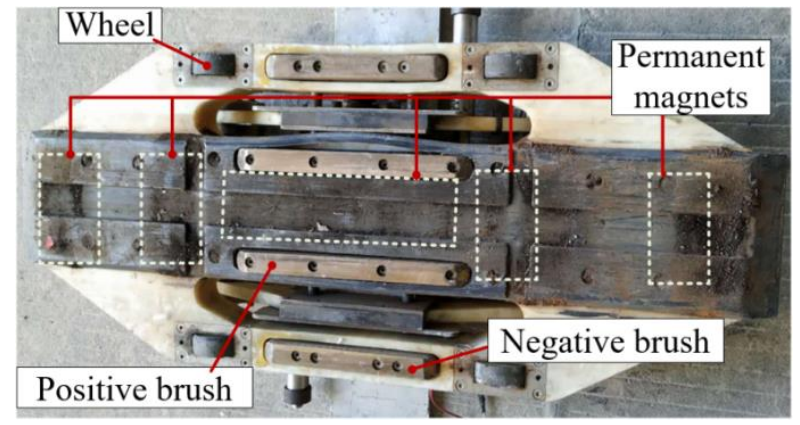

Fig. 4. The photo of the permanent magnet current collector.

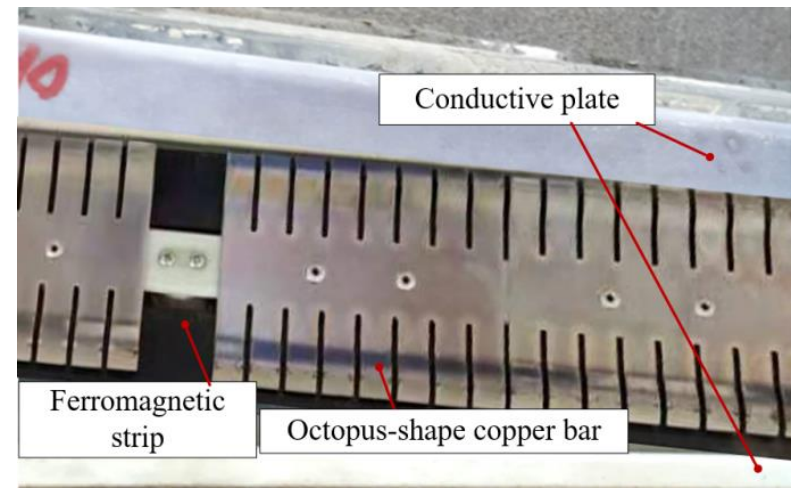

Fig. 5. The photo of the ground power supply module.

When a tram approaches a segment of the ferromagnetic strip, the partial flexible ferromagnetic strip is raised by the attractive force produced by the magnet combination in the current collector. Both the disconnected and connected states of the TCS are shown in Fig. 6. Between the ferromagnetic strip and the conducting plate, there are octopus-shaped copper sheets fixed to the ferromagnetic strip that play a key role during the power transmission process. When the copper sheets move together with the ferromagnetic strip and are driven by the attractive magnetic force to make contact with the 
conducting plate, the power supply circuit is triggered to become conducting. From this moment, electrical power is transmitted from the ground power supply module to the onboard traction and control system of the tram via the positive brush and the whole system is grounded via the negative brush. As the magnetic current collector moves away from this segment of the ferromagnetic strip, the attractive force provided by current collector becomes gradually weaker and ultimately disappears. When there is insufficient magnetic force to support the ferromagnetic strip, the strip falls and the circuit of the TCS is disconnected.

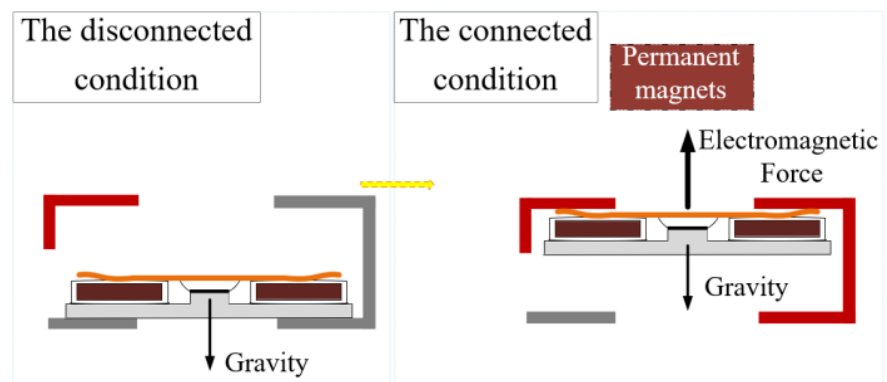

Fig. 6. The disconnected and connected conditions of the TCS.

However, experience with practical operation of the trams equipped with the TCS has shown numerous cases of severe electrochemical damage on the surface of the octopus-shape copper sheets on some segments of the ferromagnetic strips. Fig. 7(a) shows a picture of a typical damage, while the damage region is highlighted in Fig. 7(b). It can be seen that some of the tentacles of the octopus-shaped sheets are in fact completely ablated. Due to this electrochemical corrosion, the contact area between the damaged copper sheet and the conducting plate becomes smaller, which is highly likely to result in poor electrical contact in the high-voltage power supply circuit. The poor electrical contact may lead to the copper sheets suffering arc erosion more frequently, thus creating a kind of a vicious circle. Finally, a severely damaged copper sheet is unable to transfer power, which may threaten the safety and efficiency of the power transmission. In order to enhance the reliability of the TSC, it is essential to identify the causes of electrochemical corrosion in TCS.
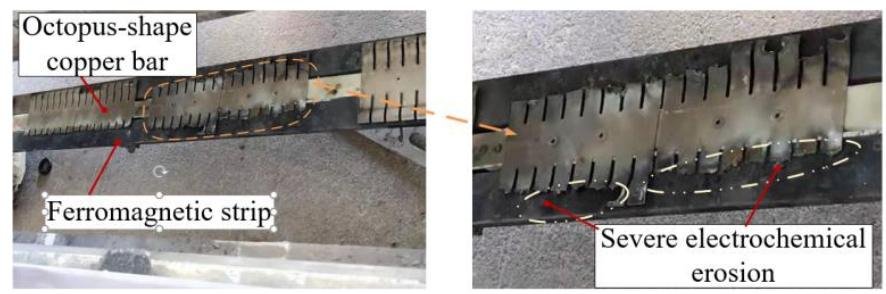

Fig. 7. The severe electrochemical corrosion observed on the surface of the octopus-shape copper sheets in the ground power supply module.

III. The Dynamic Analysis of The TCS

A. The evaluation of magnetic force between the magnetic current collector and the ferromagnetic strip
The attractive magnetic force produced by the permanent magnet combination embedded in the current collectors, placed underneath the vehicle body, raises the partial flexible ferromagnetic strips to make contact with the conducting plates. However, it has been found that as the tram accelerates the eddy currents caused by magnetic induction become unneglectable when estimating the magnetic force, especially under high-speed condition.

When evaluating the distribution of eddy currents in the segments of ferromagnetic strips, both the electric and magnetic fields are required. In regions without eddy current, only the magnetic field needs to be considered [14]. A formulation known as $\mathbf{A}, V$ - A may be used using the magnetic vector potential $\mathbf{A}$ and the electric scalar potential $V$ [15]-[17]. The field vectors are obtained from the potentials as

$$
\begin{gathered}
\mathbf{B}=\nabla \times \mathbf{A} \\
\mathbf{E}=-\frac{\partial \mathbf{A}}{\partial t}-\nabla V
\end{gathered}
$$

The governing equation for regions with eddy currents is

$$
\begin{gathered}
\nabla \times \frac{1}{\mu} \nabla \times \mathbf{A}-\nabla \frac{1}{\mu} \nabla \cdot \mathbf{A}+\sigma \frac{\partial \mathbf{A}}{\partial t}+\sigma \nabla V=\mathbf{0} \\
\nabla \cdot \sigma\left(\frac{\partial \mathbf{A}}{\partial t}+\nabla V\right)=0
\end{gathered}
$$

The governing equation for regions without eddy currents is

$$
\nabla \times \frac{1}{\mu} \nabla \cdot \mathbf{A}-\nabla \frac{1}{\mu} \nabla \cdot \mathbf{A}=\mathbf{J}_{\mathbf{S}}
$$

Where $\sigma$ and $\mu$ represent the conductivity and permeability of the conductor, respectively, and $J_{s}$ is the source current density. The boundary conditions between the regions with and without eddy currents can be defined as

$$
\begin{gathered}
\frac{1}{\mu} \nabla \cdot \mathbf{A}=0 \\
\mathbf{n} \times \mathbf{A}=\mathbf{0} \\
\mathbf{n} \cdot \mathbf{A}=\mathbf{0} \\
\frac{1}{\mu} \nabla \times \mathbf{A} \times \mathbf{n}=\mathbf{0}
\end{gathered}
$$

According to the virtual work principle, the magnetic force on an object located in a magnetic field acting along a certain direction can be calculated as

$$
f_{g}=-\frac{\partial W_{m}}{\partial g}
$$

Where $W_{m}$ is the total magnetic energy stored in the system and $g$ represents the directions along the three axis as $x, y, z$. The magnetic force only contains the components of the virtual displacement in the $x, y$ and $z$ directions.

$$
W_{m}=\frac{1}{2} \int_{\mathrm{V}_{\mathrm{m}}} \mathbf{H} \cdot \mathbf{B} d \mathrm{~V}_{m}+\frac{1}{2} \oint_{S} \mathbf{H} \times \mathbf{A} d \boldsymbol{s}
$$

$V_{m}$ represents the volume of the target area, and $S$ is the outer boundary surface of $V_{m}$.

\section{B. The TCS model for evaluating the magnetic force trends}

The procedure for assessing the trends of the magnetic force acting on the ferromagnetic strip can be split into two steps. First, for simplicity, the ferromagnetic strip can be viewed as a rigid body without flexibility for evaluating the magnetic force 
produced by the magnetic current collector in motion. Subsequently, once the dynamic distribution of the magnetic force on the ferromagnetic strip is estimated, this force distribution can be incorporated into the dynamic model of the TCS to evaluate the relative displacement between the ferromagnetic strip and the magnetic current collector, taking account of the strip flexibility.

To investigate the trends in the magnetic force of both the ferromagnetic strip and the current collector under variable speeds of the tram, a 3D finite element model was created with a mesh shown in Fig. 8. Spaces outside the strip and the collector were modelled as air regions. Four-node tetrahedron elements with a maximum side length of $12 \mathrm{~mm}$ were used throughout. The current collector moves along the static ferromagnetic strip underneath, inside which 28 permanent magnetic units are mounted. The velocity of the current collector can be adjusted between $0 \mathrm{~km} / \mathrm{h}$ and $70 \mathrm{~km} / \mathrm{h}$. The Ishaped permanent magnet combination embedded in the current collector contains 20 magnet units to provide enough magnetic force for attracting ferromagnetic strips. In addition to the I-shaped magnet combination, a 'pre-raising' magnetic module - composed of 4 magnet units - was designed to provide more magnetic force to lift the ferromagnetic strips. Two such pre-raising magnetic modules are positioned near the front and the back ends of the I-shape magnet combination, respectively. The critical parameters of a single magnet unit are listed in Table I, including some material properties of the permanent magnet units, such as relative permeability, remanence, coercivity, maximum magnetic energy product, etc. The structure and the weight of the ferromagnetic strip are fixed according in the analysis; however, it is planned to optimize both in future research. The specific settings containing the mechanical properties of the ferromagnetic strip, including Poisson's ratio, elastic modulus, and modulus of rigidity, are presented in Table I.

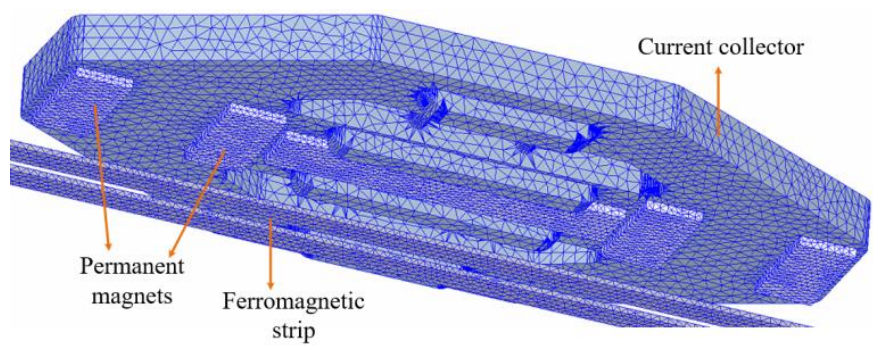

Fig. 8. Mesh configuration of the TCS model for evaluating the variation of the magnetic force distribution with changing velocity of the tram.

TABLE I

THE INITIAL SETTINGS OF CRITICAL STRUCTURAL AND MATERIAL PARAMETERS OF TCS

The key parameters of the permanent magnet combination

The size of a single permanent

magnet unit

$60 \times 30 \times 30 \mathrm{~mm}$

Density

$7740 \mathrm{~kg} / \mathrm{m}^{3}$

Remanence

$1.410 \mathrm{~T}$
Relative permeability

1.051

BH ( $\max )$

$381 \mathrm{KJ} / \mathrm{m}^{2}$

Coercivity

$1029 \mathrm{KA} / \mathrm{m}$

The key parameters of the ferromagnetic strip

$\begin{array}{cc}\text { The size of single strip section } & 27.2 \times 7.2 \times 500 \mathrm{~mm} \\ \text { Conductivity } & 2 \times 10^{6} \mathrm{~S} / \mathrm{m} \\ \text { Density } & 7872 \mathrm{~kg} / \mathrm{m}^{3} \\ \text { Poisson's ratio } & 0.25 \\ \text { Elastic modulus } & 203 \mathrm{GPa} \\ \text { Modulus of rigidity } & 79 \mathrm{GPa}\end{array}$

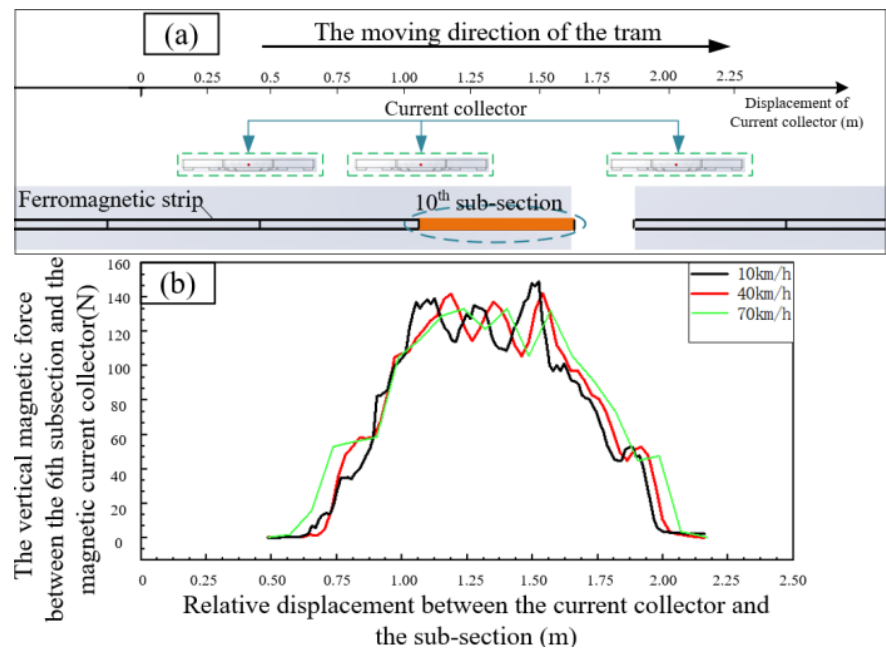

Fig. 9. (a) The dynamic process of the TCS; (b ) The magnetic force variation of a strip subsection at varying speeds.

In actual operation, the maximum speed of a tram equipped with a TCS is set to $70 \mathrm{~km} / \mathrm{h}$. The tram movement between two adjacent stations can be roughly classified into three sections: acceleration (from $0 \mathrm{~km} / \mathrm{h}$ to $70 \mathrm{~km} / \mathrm{h}$ ), uniform motion (maintaining around $70 \mathrm{~km} / \mathrm{h}$ ) and the braking/deceleration section (from $70 \mathrm{~km} / \mathrm{h}$ to $0 \mathrm{~km} / \mathrm{h}$ ). Within both the acceleration and deceleration periods, the average acceleration is measured at $1.16 \mathrm{~m} / \mathrm{s}^{2}$ approximately. If relative motion occurs between the permanent magnet and the ferromagnetic strip, eddy currents will be induced in the ferromagnetic strip. The effect of such eddy currents may gradually become quite dramatic as the relative velocity increases. The magnetic field generated by eddy currents in the strip tends to weaken the original field generated by the permanent magnets; consequently, the magnetic force produced by the permanent magnet will also be reduced. Therefore, the relative velocity is considered to be a critical variable when evaluating the trends of the magnetic force. Three typical velocities $(10 \mathrm{~km} / \mathrm{h}, 40 \mathrm{~km} / \mathrm{h}$ and $70 \mathrm{~km} / \mathrm{h})$ - which dominate at low, medium, and high speed levels, respectively - have been chosen. When evaluating the force distribution on the ferromagnetic strip produced by the magnetic current collector, the flexibility of the ferromagnetic strip was initially not considered. When calculating the 
magnetic force, an individual segment of the strip (with the standard length of $5 \mathrm{~m}$ ) was divided into 10 uniform subsections of $0.5 \mathrm{~m}$ length. Each subsection experiences the same magnetic force variation tendency when relative motion occurs between the strip and the current collector for a given speed. The variation of the magnetic force for one subsection is shown in Fig. 9(b) for a situation depicted in Fig. 9(a).

TABLE II

THE PERFoRMANCE INDICATORS OF THE FERROMAGNETIC STRIP WITH DIFFERENT SPEEDS OF THE CURRENT COLLECTOR

\begin{tabular}{cccc}
\hline \multicolumn{4}{c}{ DIFFERENT SPEEDS OF THE CURRENT COLLECTOR } \\
& $\begin{array}{c}\text { Case } 1 \\
(10 \mathrm{~km} / \mathrm{h})\end{array}$ & $\begin{array}{c}\text { Case2 } \\
(40 \mathrm{~km} / \mathrm{h})\end{array}$ & $\begin{array}{c}\text { Case3 } \\
(70 \mathrm{~km} / \mathrm{h})\end{array}$ \\
\hline $\begin{array}{c}\text { The maximum of } \\
\text { flux density }\end{array}$ & $2.08 \mathrm{~T}$ & $2.05 \mathrm{~T}$ & $1.99 \mathrm{~T}$ \\
$\begin{array}{c}\text { The maximum of } \\
\text { eddy current density } \\
\begin{array}{c}\text { Maximum magnetic } \\
\text { force }\end{array}\end{array}$ & $2.67 \mathrm{e} 5$ & $6.44 \mathrm{e} 5$ & $1.56 \mathrm{e} 6$ \\
$\left(\mathrm{~A} / \mathrm{m}^{2}\right)$ & $\left(\mathrm{A} / \mathrm{m}^{2}\right)$ & $\left(\mathrm{A} / \mathrm{m}^{2}\right)$ \\
\hline \hline
\end{tabular}

As shown in Fig. 9(b), the peak value of the magnetic force on the $10^{\text {th }}$ subsection is $148 \mathrm{~N}$ when the speed of the current collector reaches $10 \mathrm{~km} / \mathrm{h}$. Three different velocities have been used to observe the impact on the force brought by eddy currents induced in the strips. An interesting phenomenon is observed that when the tram speed increases from $10 \mathrm{~km} / \mathrm{h}$ to $70 \mathrm{~km} / \mathrm{h}$, as shown in Table II, the maximum current in the ferromagnetic strip rises from $2.67 \mathrm{e} 5\left(\mathrm{~A} / \mathrm{m}^{2}\right)$ to $1.56 \mathrm{e} 6\left(\mathrm{~A} / \mathrm{m}^{2}\right)$, hence by a factor of five, whereas the maximum of flux density drops from $2.08 \mathrm{~T}$ to $1.99 \mathrm{~T}$, a decrease of $4.3 \%$. The magnetic force decreases from $148 \mathrm{~N}$ to $135 \mathrm{~N}$ (by 8.78\%) when the velocity increases from $10 \mathrm{~km} / \mathrm{h}$ to $70 \mathrm{~km} / \mathrm{h}$. The varying trends of the magnetic force provide the foundation for analyzing the dynamic character of the TCS when considering the flexibility of the ferromagnetic strips.

\section{The dynamic analysis of the TCS considering the flexibility of ferromagnetic strips}

In order to analyze the deformation process of the flexible ferromagnetic strips under the action of the magnetic force, the magnetic force variation of the selected strip segment obtained in previous section - was fed into the dynamic model of the TCS. From this model, the connecting and disconnecting processes between the conducting plate and the ferromagnetic strip - taking account of its flexibility - were simulated at the speed of $10 \mathrm{~km} / \mathrm{h}$ and are presented in Fig. 10. The air gap between the strip and the conductive plate is $28 \mathrm{~mm}$, without the action of the magnetic force. When the strip is raised by 28 $\mathrm{mm}$ by magnetic attraction, it makes contact with the conducting plate and the power supply is switched on.

The whole displacement process of a flexible ferromagnetic strip segment under the action of the moving magnetic current collector is presented in a series of graphs in Fig. 11, at the speed of $10 \mathrm{~km} / \mathrm{h}$. As the current collector moves relative to the strip segment, a typical case of complete contact between the strip and the conducting plate is shown in Fig. 11(b). The partial flexible ferromagnetic strip is raised up by $28 \mathrm{~mm}$.
However, when the current collector is at the front or the back end of the strip segment, poor contacts appear as in Fig. 11(a) and Fig. 11(c), respectively. Although the end of the strip is raised to some extent, the vertical displacement does not reach $28 \mathrm{~mm}$.

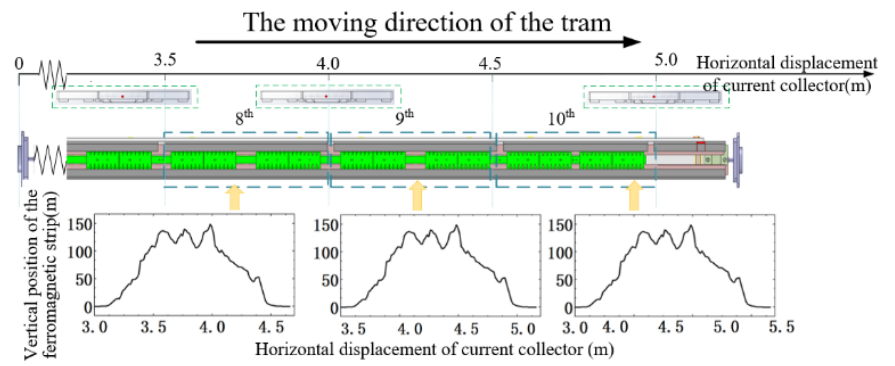

Fig. 10. The dynamic process simulation of the TCS taking account of the flexibility of ferromagnetic strips.

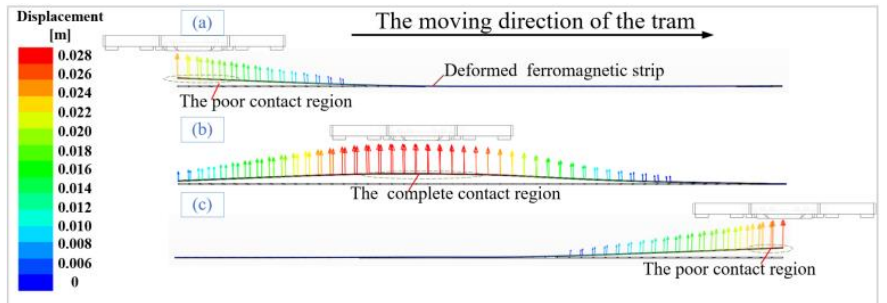

Fig. 11. The displacement of the flexible ferromagnetic strip in TCS during the dynamic process.

With the consideration of the balance between the magnetic force of the ferromagnetic strip and its gravity, the motion trajectory of the $10^{\text {th }}$ subsection in the whole strip segment is presented in Fig. 12(a), with the relative motion velocity between the magnetic collector and the ground power supply system set to $10 \mathrm{~km} / \mathrm{h}$. In addition to the displacement of the current collector relative to $10^{\text {th }}$ subsection, it is worth noting that some vibration happens during the conducting time zone of the power supply circuit. When the current collector moves roughly $4.75 \mathrm{~m}$ relative to the $10^{\text {th }}$ subsection of the strip, these vibrations take place, which may indicate poor electrical contact and even trigger arc erosion. The poor contact between the ferromagnetic strip and the conducting plate - which can be viewed as an 'off-line' case - may happen when the magnetic force produced by the current collector cannot support the weight of the strip subsections. The off-line case shows the contact state between the strip and the conducting plate, which is suitable for analyzing the off-line process of the system. The duration of the arc erosion can be evaluated via this off-line duration when the power supply is still in the state of conducting. The good and poor contact durations of the whole circuit are highlighted in Fig. 12(b). The off-line duration within the conducting time zone of the circuit lasts $0.122 \mathrm{~s}$, in which the largest air gap between the strip and conductive plate is $3.40 \mathrm{~mm}$. During the disconnection process, the strip falls with the average speed of $2 \mathrm{~cm} / \mathrm{s}$ as the magnetic force weakens. The poor contact between the strip and the conducting plate is also illustrated in Fig. 13. 


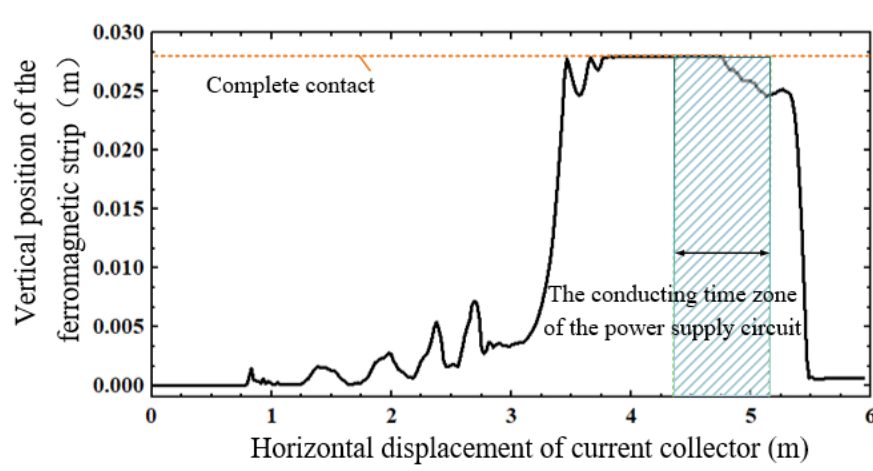

(a)

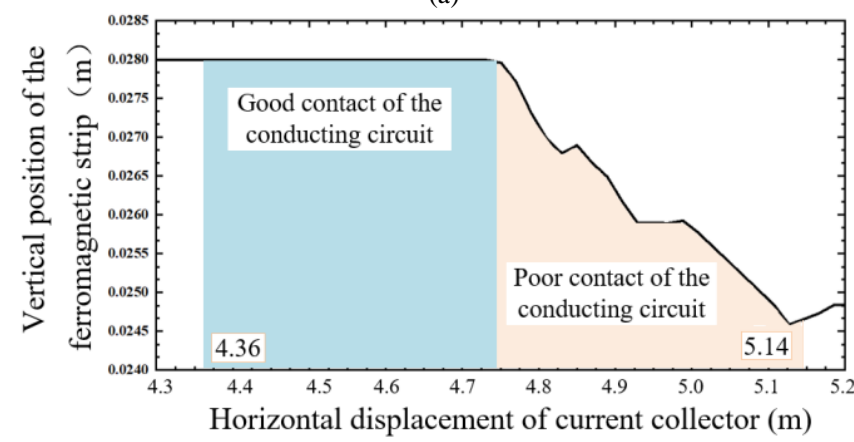

(b)

Fig. 12. (a) Horizontal displacement of the current collector relative to the 10th subsection; (b) Conducting time zone of the power supply circuit.

The dynamic conditions, with three different velocities of $10 \mathrm{~km} / \mathrm{h}, 40 \mathrm{~km} / \mathrm{h}$ and $70 \mathrm{~km} / \mathrm{h}$ have been tried and some of the critical performance indicators are listed in Table III. During the falling process of the partial ferromagnetic strip, the varying-speed deformation of the ferromagnetic strip is determined by the dynamic balance between the magnetic force and its weight. When the tram velocity increases from 10 $\mathrm{km} / \mathrm{h}$ to $70 \mathrm{~km} / \mathrm{h}$, the maximum magnetic force on the partial ferromagnetic strip decreases from $148 \mathrm{~N}$ to $135 \mathrm{~N}$ (by 8.78\%) due to the influence of eddy currents. However, the off-line duration is shortened dramatically from $122 \mathrm{~ms}$ to $14 \mathrm{~ms}$ (by $88.52 \%$ ). Compared with the limited impact brought by the eddy currents, the off-line duration between the ferromagnetic strip and the conductive plate is mainly influenced by the increasing velocity of the tram. The severity of electrochemical erosion emerging in the power supply modules tends to be mitigated somewhat as the speed of the tram increases.

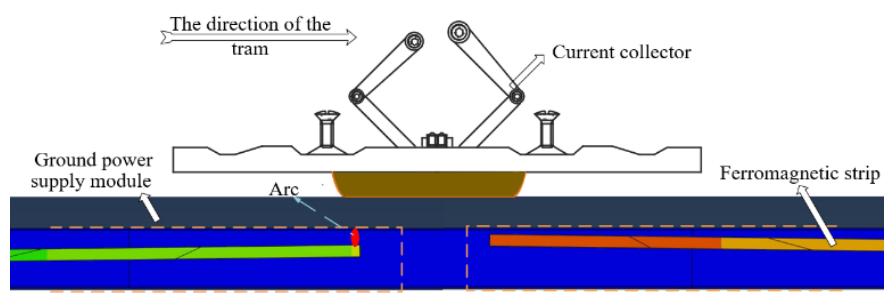

Fig.13. Poor contact between the strip and the conducting plate.
TABLE III

THE PERFORMANCE INDICATORS DURING THE DEFORMATION PROCESS OF THE FERROMAGNETIC STRIP WITH DIFFERENT SPEEDS

\begin{tabular}{cccc}
\hline & $\begin{array}{c}\text { Case1 } \\
(10 \mathrm{~km} / \mathrm{h})\end{array}$ & $\begin{array}{c}\text { Case2 } \\
(40 \mathrm{~km} / \mathrm{h})\end{array}$ & $\begin{array}{c}\text { Case3 } \\
(70 \mathrm{~km} / \mathrm{h})\end{array}$ \\
\hline $\begin{array}{c}\text { Maximum } \\
\text { magnetic force } \\
\text { Off-line duration } \\
\begin{array}{c}\text { The average falling } \\
\text { speed of the } \\
\text { ferromagnetic strip }\end{array}\end{array}$ & $148 \mathrm{~N}$ & $142 \mathrm{~N}$ & $135 \mathrm{~N}$ \\
\hline \hline
\end{tabular}

\section{THE ARC ERosion EXPERIMENTS AND FIELD INVESTIGATION}

\section{A. The Arc Erosion Experiments}

Based on the dynamic model of the TCS, the displacements of the flexible ferromagnetic strip both during the connecting and disconnecting phases between the strip and the conducting plate have been considered. To evaluate the electrochemical damage of copper sheets due to the motion, a series of arc discharge experiments have been performed. The experimental platform used to observe the phenomenon of arc discharge is shown in Fig. 14. The arc with adjustable air gap between the positive and negative electrodes is driven by a servo motor, which can simulate the disconnecting process between the copper sheet and the conducting plate. A direct-current power source (voltage $120 \mathrm{~V}$, current $10 \mathrm{~A}$ ) was used. As the magnetic current collector gets further away from the segment of the ferromagnetic strip, three cases with velocities of 10 $\mathrm{km} / \mathrm{h}, 40 \mathrm{~km} / \mathrm{h}$ and $70 \mathrm{~km} / \mathrm{h}$ were attempted. These three cases correspond to the disconnecting conditions under different relative velocities $(2 \mathrm{~cm} / \mathrm{s}, 6 \mathrm{~cm} / \mathrm{s}$ and $10 \mathrm{~cm} / \mathrm{s})$ between the ferromagnetic strip and conductive plate. The dynamic simulation model of the relative motion between the copper sheet and plate conducting plate was used to guide the settings for the experiments.

The worst case of arc damage was found when the relative velocity was set to $2 \mathrm{~cm} / \mathrm{s}$. The variation tendency of the arc diameter indicates the development of the arc. As shown in Fig. 15, the whole arc development consists of four phases: arc igniting, arc diffusing, arc burning steadily and arc extinguishing. A series of typical snapshots during these four different phases have been recorded by a high-speed camera and are shown in Fig. 16.

At ignition, the arc occurs when the copper sheet starts separating from the anode from 0 to $20 \mathrm{~ms}$. From $20 \mathrm{~ms}$ to 150 $\mathrm{ms}$, as the distance between the copper sheet and the anode increases, the diameter of the arc increases constantly from $0.527 \mathrm{~mm}$ to $2.228 \mathrm{~mm}$ with the rate of increase of 0.0131 $\mathrm{mm} / \mathrm{ms}$. Compared with the arc diffusing phase, the diameter of the arc increases slower from $2.228 \mathrm{~mm}$ to $2.458 \mathrm{~mm}$, with the comparatively lower rate of $0.0029 \mathrm{~mm} / \mathrm{ms}$, until the arc is burning steadily (from $150 \mathrm{~ms}$ to $230 \mathrm{~ms}$ ). 


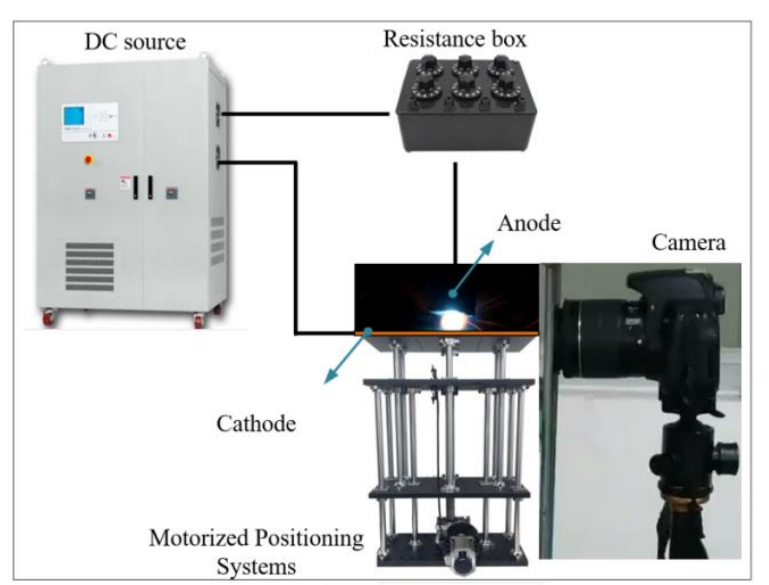

Fig. 14. The arc discharge experimental platform.

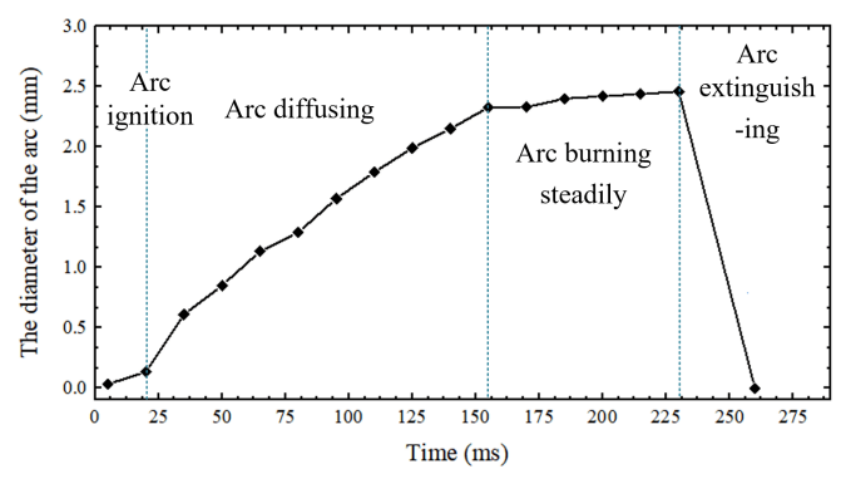

Fig. 15. The record of arc diameter variation during the discharge process.

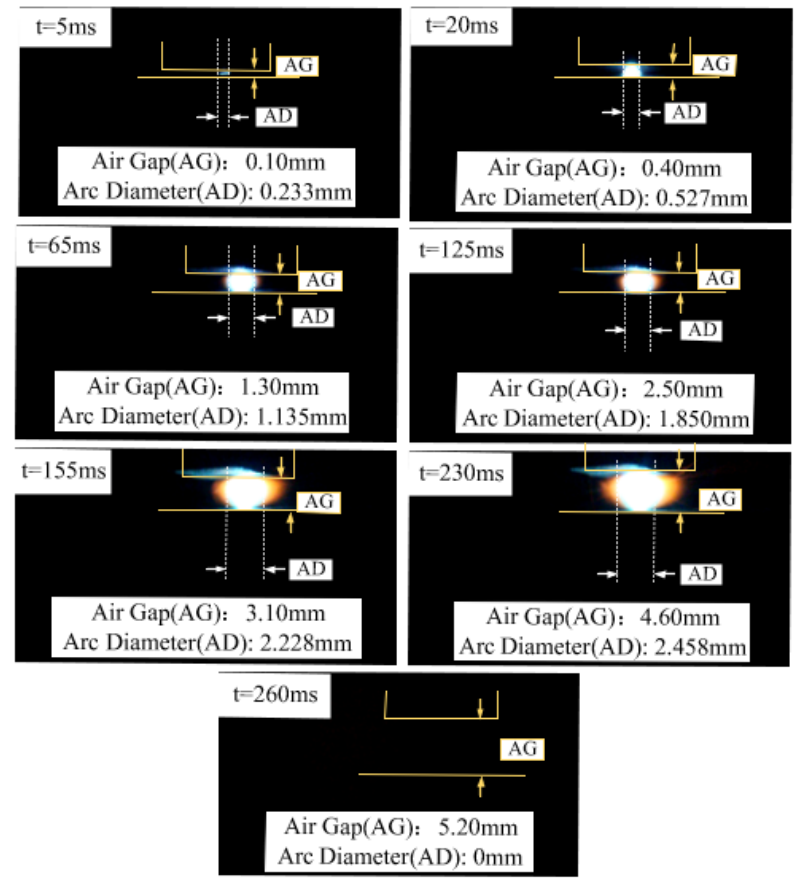

Fig. 16. The images of arc development during arc discharge process.

The area of the erosion trace on the surface of the copper sheets, at the three disconnecting speeds, are highlighted in Fig. 17. Details of the tests are listed in Table IV. It can be seen that when the speed of the tram is $10 \mathrm{~km} / \mathrm{h}$, the area of the erosion at $2.103 \mathrm{~mm}^{2}$ is more than twice bigger than for the case of 40 $\mathrm{km} / \mathrm{h}$ and is about three times larger than for the case of 70 $\mathrm{km} / \mathrm{h}$. Based on these erosion traces, the electrochemical erosion is more severe at the lower speeds of trams.

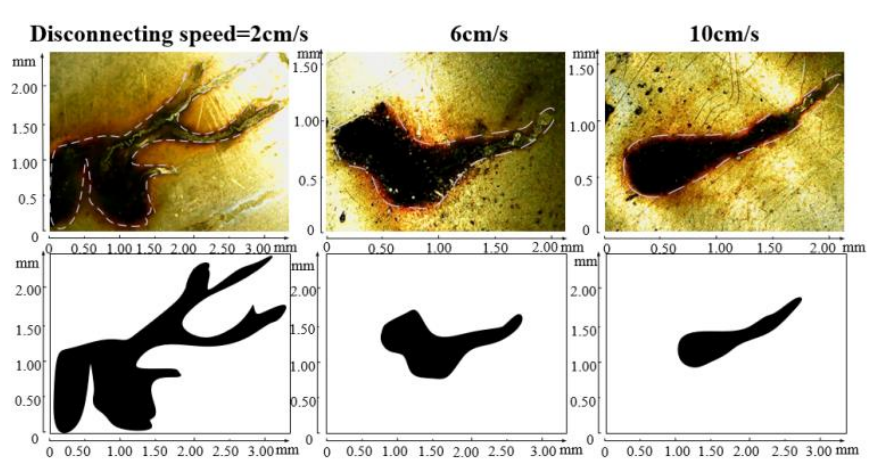

Fig. 17. The electrochemical erosion trace with different speeds of trams

TABLE IV

The AREa of ERosion Traces With DiFFERENT Velocities BeTweEN ANODE AND CATHODE

\begin{tabular}{lccc}
\hline \hline & $\begin{array}{c}\text { Case } 1 \\
(10 \mathrm{~km} / \mathrm{h})\end{array}$ & $\begin{array}{c}\text { Case } 2 \\
(40 \mathrm{~km} / \mathrm{h})\end{array}$ & $\begin{array}{c}\text { Case } 3 \\
(70 \mathrm{~km} / \mathrm{h})\end{array}$ \\
\hline $\begin{array}{l}\text { The disconnecting velocities } \\
\text { between anode and cathode }\end{array}$ & $2 \mathrm{~cm} / \mathrm{s}$ & $6 \mathrm{~cm} / \mathrm{s}$ & $10 \mathrm{~cm} / \mathrm{s}$ \\
The area of erosion traces & $2.103 \mathrm{~mm}^{2}$ & $0.918 \mathrm{~mm}^{2}$ & $0.673 \mathrm{~mm}^{2}$ \\
\hline \hline
\end{tabular}

\section{B. Field investigations of the cases of damage to TCSs}

Following on from the dynamic simulations and relevant arc experiments, the damage trend was established, namely that with the tram speeds increasing the period of off-line duration is reduced while the severity of arc erosion in TCSs tends to become gradually less. To verify the validity of these damage development trends, some field investigations of damage to the TCSs have been undertaken and the corresponding statistics are presented in Fig. 18.

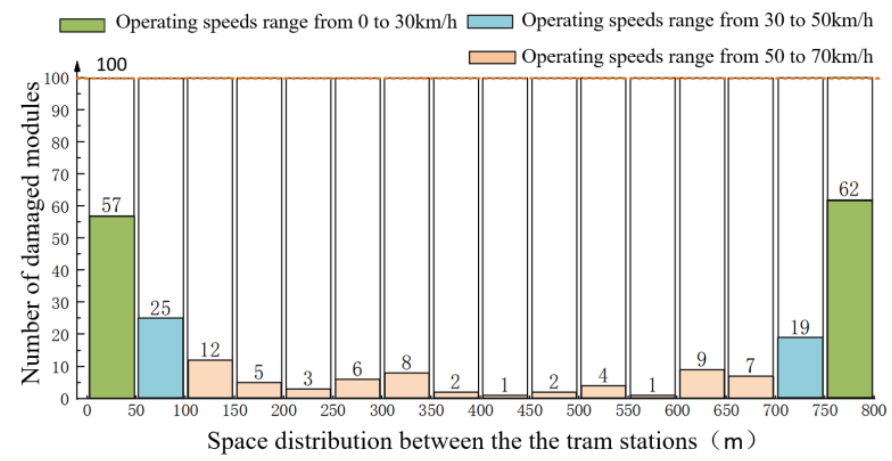

Fig.18. The statistical information about damage to TCSs.

The distance between two adjacent stations is approximately $0.8 \mathrm{~km}$, which - for the purpose of this analysis - is split into 16 sub-sections, each of which has 100 segments of ground power supply modules, as depicted in Fig. 18. It can be seen that in this practical survey the number of damaged power supply modules in the low-speed region close to the stations is dramatically larger than for the medium or high-speed regions, which are inevitably located relatively far away from the 
stations. The main cases of damage are in fact concentrated in the regions with the speeds between $0 \mathrm{~km} / \mathrm{h}$ to $30 \mathrm{~km} / \mathrm{h}$. This is consistent with the previous findings based on simulation.

To alleviate the issue of damage due to electrochemical corrosion, the structure of the permanent magnetic modules is planned to be optimized to provide more magnetic force with better distribution, while the acceleration performance of motors on the trams could be improved to shorten the acceleration zone to reduce the damage risk of the TCS.

\section{CONCLUSION}

As a novel power supply system, the Tramwave Catenaryfree System (TCS) has been used to provide constant and stable electric power for urban traffic in many countries over the world. However, during the operation of TCS some serious electrochemical corrosion cases have been exposed in the ground power supply modules of TCSs, which directly impact the operational safety of the trams.

In order to identify the causes leading to these damages in TCS, a transient FEM model of TCS was built to evaluate the magnetic force provided by the magnetic current collector. The variation of the magnetic force was then incorporated into a dynamic model of the TCS allowing for the material flexibility of the ferromagnetic strip. The deformation processes of the flexible ferromagnetic strip under the action of the magnetic current collector with different velocities of trams were accomplished using this dynamic model.

Through a series of tests, it was found that the off-line duration between the partial ferromagnetic strip and the conducting plate within the conducting time zone of the whole power supply circuit was indicative of the quality of the electrical contact between them. Ultimately, it was found that the impact brought by the eddy currents on the off-line duration was relatively limited. The off-line duration, which directly correlates with the severity of electrochemical erosion, is mainly determined by the velocity of the tram. The risk of suffering the electrochemical erosion decreases as the speed of the tram rises, which has also been verified by the practical survey of the damage cases for a particular tram line using the TCS. The conclusions from this study will provide foundation for further optimization of the system to alleviate the electrochemical erosion issues. In our future research, the structural optimization of the permanent magnets and ferromagnetic strip and/or adding controllable electromagnets will be considered to mitigate the electrochemical corrosion issues and improve the power supply quality of the Tramwave Catenary-free Power Supply System.

\section{REFERENCES}

[1] L. Cheng, P. Acuna, S. Wei, J. Fletcher, W. Wang and J. Jiang, "FastSwap charging: an improved operation mode for catenary-free light rail networks," IEEE Trans. Veh. Technol., vol. 67, no. 4, pp. 2912-2920, 2018.

[2] H. Al-Ezee, S. B. Tennakoon, I. Taylor and D. Scheidecker, "Aspects of catenary free operation of DC traction systems," in Proc. 50th Int. Univ.
Power Eng. Conf. (UPEC), Stoke on Trent, United Kingdom, 2015, pp. $1-5$.

[3] L. Pastena, "A catenary-free electrification for urban transport: an overview of the tramwave system," IEEE Elect. Mag., vol. 2, no. 3, pp. 16-21, 2014.

[4] S. Midya, D. Bormann, T. Schutte and R. Thottappillil, "Pantograph arcing in electrified railways - mechanism and influence of various parameters-part I: with DC traction power supply," IEEE Trans. Power Del., vol. 24, no. 4, pp. 1931-1939, 2009.

[5] Z. Yang, P. Xu, W. Wei, G. Gao, N. Zhou and G. Wu, "Influence of the Crosswind on the Pantograph Arcing Dynamics," IEEE Trans. Plasma Sci., vol. 48, no. 8, pp. 2822-2830, 2020.

[6] G. Gao, J. Hao, W. Wei, H. Hu, G. Zhu and G. Wu, "Dynamics of pantograph-catenary arc during the pantograph lowering process," IEEE Trans. Plasma Sci., vol. 44, no. 11, pp. 2715-2723, 2016.

[7] Y. Kubota, T. Hayasaka, T. Miyauchi, H. Nozaki, T. Hirai, and T. Matsumoto, "Effect of arc discharge on the wear profile of $\mathrm{Cu}$ impregnated carbon based pantograph contact strip," in Proc. 27th Int. Conf. Elect. Contacts (ICEC), Deutschland, Dresden, 2014, pp. 1-6.

[8] Y. Song, Z. Liu, H. Wang, et al. "Nonlinear analysis of wind-induced vibration of high-speed railway catenary and its influence on pantograph-catenary interaction," Vehicle Syst Dyn., vol. 54, no. 6, pp. 723-747, 2016.

[9] S. Midya, D. Bormann, T. Schütte and R. Thottappillil, "DC component from pantograph arcing in AC traction system-influencing parameters, impact, and mitigation techniques," IEEE Trans. Electromagn. Compat., vol. 53, no. 1, pp. 18-27, 2011.

[10] O. Wang, A. Núñez, et al. "Analysis of the evolvement of contact wire wear irregularity in railway catenary based on historical data," Vehicle Syst Dyn., vol. 56, no. 8, pp. 1207-1232, 2018.

[11] S. Yang, et al. "Nonlinear modelling of high-speed catenary based on analytical expressions of cable and truss elements," Vehicle Syst Dyn., pp. 1-25, 2015.

[12] S. Lee, J. Bae and W. Kim, "Design Process of Spoke-Type Permanent Magnet Synchronous Motor Considering Magnetization Performance," IEEE Trans. Appl. Supercond., vol. 30, no. 4, pp. 1-6, 2020.

[13] A. Bermúdez, A. L. Rodríguez and I. Villar, "Extended formulas to compute resultant and contact electromagnetic force and torque from Maxwell stress tensors," IEEE Trans. Magn., vol. 53, no. 4, pp. 1-9, 2017.

[14] M. Messadi, "Eddy Current Computation in Translational Motion Conductive Plate of an Induction Heater With Consideration of Finite Length Extremity Effects," IEEE Trans. Magn., vol. 52, no. 3, pp. 1-4, 2016.

[15] L. Lombardi, D. Romano and G. Antonini, "Analytical Formula for the Magnetic-to-Electric Field Coupling of Magnetization in the Partial Element Equivalent Circuit Method," IEEE Trans. Magn., vol. 54, no. 10, pp. 1-12, 2018.

[16] J. McDonald, K. Hsieh and S. Satapathy, "Edge Elements and Current Diffusion,” IEEE Trans. Plasma Sci., vol. 39, no. 1, pp. 437-441, 2011.

[17] M. Moghaddami, A. I. Sarwat and F. de Leon, "Reduction of Stray Loss in Power Transformers Using Horizontal Magnetic Wall Shunts," IEEE Trans. Magn., vol. 53, no. 2, pp. 1-7, 2017. 


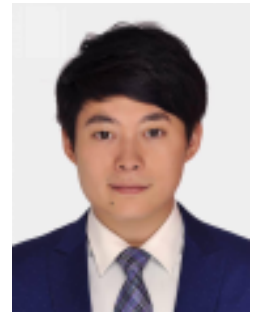

Song Xiao received the B.Sc. and M. Sc. Degree in Electrical Engineering and Automation from Chengdu University of Technology, and his Ph.D. degree in electrical and electronic engineering from University of Southampton, UK, in 2014.

$\mathrm{He}$ is currently an Associate Professor with the School of Electrical Engineering, Southwest Jiaotong University, Chengdu, China. His current research interests include electrical equipment stability, electrical materials complex electro- magnetic characteristics measurement, optimization design theory and application research.

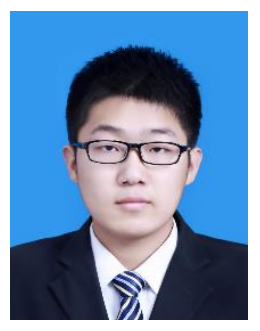

Can Zhang was born in Anhui, China, in 1996. He is currently pursuing the M.Sc. degree with the School of Electrical Engineering, Southwest Jiaotong University, Chengdu, China. His current research interests include the electrochemical corrosion of electrified transit system for urban railway.

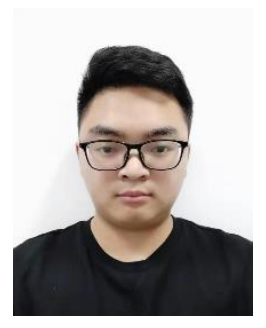

Yuanpei Luo was born in Sichuan, China, in 1996. He is currently pursuing the M.Sc. degree with the School of Electrical Engineering, Southwest Jiaotong University, Chengdu, China. His current research interests include the simulation and experimental study of the new pantograph-catenary system.

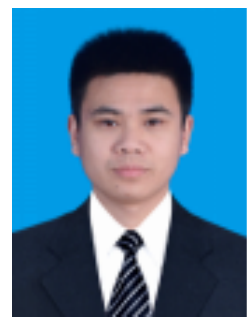

Jingchi Wu was born in Sichuan, China, in 1995. He is currently pursuing the M.Sc. degree with the School of Electrical Engineering, Southwest Jiaotong University, Chengdu, China. His current research interests include the suspension system of maglev train and the frequency analysis of power system.

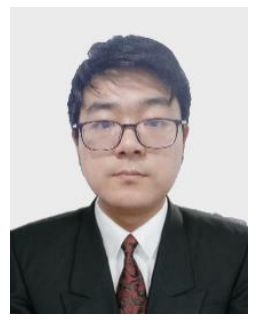

Yang Rao was born in Jiangxi, China, in 1997. He is currently pursuing the M.Sc. degree with the School of Electrical Engineering, Southwest Jiaotong University, Chengdu, China. His current research interests include the overvoltage analysis and protection on switching transients in high speed trains.

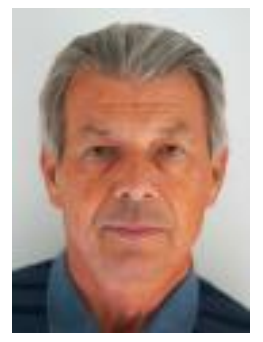

Jan K. Sykulski is currently a Professor of Applied Electromagnetics at the University of Southampton, UK. He is founding Secretary of International Compumag Society. He is a Fellow of IEEE, a Fellow of IET, a Fellow of the Institute of Physics and a Fellow of the British Computer Society.

His personal research is in the development of fundamental methods of computational electromagnetics, simulation of coupled field systems and design and optimization of electromechanical devices. 